Infusionstherapie 1990;17:288

\title{
Inhalt, Vol. 17, No. 6, 1990
}

\section{Contents}

Übersichtsarbeiten

Richter, G.; Dehnert, J. Sondenernährung in der Onkologie

291

Originalarbeiten

Günther, H.J.; Saeger, H.D.; Hohner, E.; Striebel. J.P.;

Scigalla, P.; Bartels, E; Reichardt, I.

Peripher-venöse bedarfsadapt ${ }_{j}$ erte parenterale

Ernährung: Renaissance der Maltose?

300

Druml, W.; Zadravec, S.; Kerbl, H.; Grimm, G.;

Schneeweiß, B.

Intensivmedizinischer Einsatz einer neuen Fettemulsion . 306

Penner, M.; Fingerhut, D.; Tacke, A.

Einfluß einer neuen Hydroxyäthylstärkelösung

10\% HES/270/0,5 auf Blutgerinnung, Blutverlust und

Hämodynamik im Vergleich zu PPL 3,5 \% 314

Baak, L.C.; J arisen, J.B.M.J.; Lamers, C.B.H.W.

Effekt von intravenösen Aminosäuren auf intragastrale

pH-Werte während ambulant er pH-Metrie 321

ABSTRACTS der 22. Jahrestagung der Deutschen

Arbeitsgemeinschaft für Histokompatibilitätstestung,

Hamburg, 4.-6. Oktober 1990326

Informationen für die Klinik

Ausschuß für Nährstoffbedarf der DAKE:

L-Tryptophan in Infusionslösungen 352

Mitteilungen der Deutschen Gesellschaft für Transfusionsmedizin und Immunhämatologie (DGTI) . . 353

Editorial 289

Impressum 287

Inhalt 288

Buchbesprechungen

Autorenverzeichnis 1990

Jahresinhalt 1990

Sachwortverzeichnis

Hinweise für Autoren

Review Articles

Richter, G.; Dehnert, J. 
Enteral Tube Feeding in Clinical Oncology 291

Original Papers

Günther, H.J.; Saeger, H.D.; Hohner, E.; Striebel. J.P.;

Scigalla, P.; Bartels, E; Reichardt, I.

A Peripheral-Venous Requirement-adapted Parenteral

Nutrition: Renaissance of Maltose? 300

Druml, W.; Zadravec, S.; Kerbl, H.; Grimm, G.;

Schneeweiß, B.

Parenteral Nutrition in Intensive Care Patients:

Experiences with a New Fat Emulsion 306

Penner, M.; Fingerhut, D.; Tacke, A.

Influence of a New Hydroxyethylstarch Preparation

(10\% HES/270/0.5) on Hemostasis, Blood-loss and

Hemodynamics Compared to Plasma Protein Solution . . 314

Baak, L.C.; Jansen, J.B.M.J.; Lamers, C.B.H.W.

The Effect of Intravenous Amino Acids on Intragastric

$\mathrm{pH}$ During Continuous Intragastric $\mathrm{pH}$-Monitoring.... 321

ABSTRACTS -22. Annual Meeting of the Deutsche

Arbeitsgemeinschaft für Histokompatibilitätstestung,

Hamburg, October, 4-6,1990 326

Clinical Information

Committee for nutrient requirements of the DAKE:

L-Tryptophan in Infusion Solutions 352

Report of the Deutsche Gesellschaft für

Transfusionsmedizin und Immunhämatologie (DGTI) . . 353

Editorial 289

Imprint $\quad 287$

Contents 288

Book Reviews 352

Author Index 1990

Complete Contents $1990 \quad 359$

Subject Index 363

Instructions to Authors 364

Bibliographischer Hinweis: Inhaltsverzeichnisse dieser Zeitschrift erscheinen regelmäßig in current contents ${ }^{\circledR}$ sowie in anderen bibliographischen Diensten. 